\title{
Labyrinthe
}

$7 \mid 2000$

Numéro 7

\section{La Confédération des Étudiants-Chercheurs}

\section{Laurence Marie}

\section{OpenEdition}

\section{Journals}

Édition électronique

URL : http://journals.openedition.org/labyrinthe/725

DOI : 10.4000/labyrinthe.725

ISSN : 1950-6031

Éditeur

Hermann

Édition imprimée

Date de publication : 30 octobre 2000

Pagination : 177-179

Référence électronique

Laurence Marie, «La Confédération des Étudiants-Chercheurs », Labyrinthe [En ligne], 7 | 2000, mis en ligne le 20 avril 2005, consulté le 20 avril 2019. URL : http://journals.openedition.org/labyrinthe/725 ; DOI : 10.4000/labyrinthe.725

Ce document a été généré automatiquement le 20 avril 2019

Propriété intellectuelle 


\title{
La Confédération des Étudiants- Chercheurs
}

\author{
Laurence Marie
}

1 Comment gérer les problèmes rencontrés pendant sa thèse ou après la soutenance ? C'est la question à laquelle essaient de répondre les membres de la Confédération des Étudiants-Chercheurs. Structure interdisciplinaire, indépendante des syndicats traditionnels et des partis politiques, l'association a pour ambition de fédérer les efforts accomplis à un niveau local par les associations de doctorants.

Décembre 1994 : des étudiants-chercheurs créent HotDocs, forum de discussion sur internet entre étudiants d'horizons différents.

3 Mai 1995 : le mouvement HotDocs publie le rapport « Formation doctorale : enjeux, bilans, propositions ", recensant les différents problèmes rencontrés par les doctorants au cours de leur thèse et après leur soutenance : financement de la thèse, encadrement parfois trop lâche du doctorant, harmonisation de la durée des thèses et responsabilisation des différentes parties prenantes (doctorant, directeur de thèse, directeur du laboratoire, directeur de l'école doctorale) sur les droits et les devoirs de chacun.

4 Mars 1996 : la Confédération des Étudiants-Chercheurs (CEC) est formée afin de fédérer les efforts accomplis à un niveau local ou dans certaines disciplines. Regroupant plus de deux mille doctorants issus de toutes les disciplines universitaires, elle se veut une force de proposition pour le troisième cycle, l'enseignement supérieur et les études doctorales et affiche deux objectifs principaux : «obtenir la reconnaissance d'un véritable statut pour les doctorants ; améliorer au niveau national l'adéquation entre le doctorant et le marché du travail, en contribuant notamment au développement des débouchés professionnels tant publics que privés ".

51998 : Après de nombreuses tables rondes, une première victoire est remportée à travers la mise en place obligatoire d'une charte des thèses dans toutes les universités.

Cependant, les membres de la CEC veulent aller plus loin et voir adopter un véritable Contrat de Thèse à valeur juridique, afin que les doctorants se voient garantir un statut. « Nous voulons que soit reconnue l'existence d'un troisième corps qui n'est ni usager, ni 
personnel de l'université, mais un mélange des deux », explique Guillaume Bonello, viceprésident de l'association, en thèse d'astrophysique instrumentale à Orsay. En attendant, les membres de la CEC concentrent leurs efforts sur la mise en place d'un observatoire des thèses, et sur la revalorisation de l'allocation de recherche, dont le montant inchangé depuis dix ans approche maintenant le SMIC.

\section{http ://garp. univ-bpclermont. fr/cec}

Principales dispositions du contrat de thèse proposé par la CEC :

Rendre le financement obligatoire. Un doctorant n'est plus un étudiant qui acquiert un savoir : son rôle est de produire du savoir. Il doit donc être salarié et bénéficier des dispositions sociales en vigueur. Ce financement peut prendre plusieurs formes, selon que le doctorant soit en formation initiale [...] ou continue [...].

Renforcer l'encadrement de la thèse. Le directeur de thèse ne peut encadrer plus de deux doctorants simultanément. Il est mis fin à la séparation artificielle entre directeur " officiel ", titulaire d'une habilitation à diriger des recherches, et encadrant effectif. Il est en effet possible à un chercheur non habilité d'obtenir une dérogation pour diriger officiellement le doctorant qu'il encadre en pratique.

Assurer la médiation des conflits. Le doctorant a la possibilité, en cas de conflits avec son directeur de thèse, d'en référer à des médiateurs (l'un dans l'université dont il dépend, l'autre au niveau national dans la section du CNU correspondant à sa discipline).

Assurer la qualité des thèses soutenues. L'autorisation de soutenance n'est accordée que si le doctorant a au moins une publication à son actif.

Fournir un document de référence. Après la soutenance, le $\mathrm{CdT}$ sert au jeune docteur de descriptif de sa formation : travail de recherche, formations complémentaires, etc. En effet, la formation doctorale ne se réduit pas à la production d'un document appelé " thèse ", elle comporte aussi un volet « acquisition de compétences " qui est à valoriser.

Unifier la durée des thèses. La thèse est limitée à trois ans en formation initiale, quatre ans en formation continue. Des dérogations clairement justifiées peuvent être demandées pour une année supplémentaire.

Renforcer le rôle du directeur de formation doctorale et particulièrement d'école doctorale. Il est garant du bon déroulement et de la qualité des thèses depuis le choix du sujet jusqu'à la délivrance du diplôme.

15 Assurer le suivi de la formation doctorale. Des statistiques sont tenues pour connaître le devenir des doctorants et des jeunes docteurs. Elles seraient établies sur le modèle des enquêtes de la DGRT afin de faciliter la comparaison avec les données nationales. Elles servent aux différentes instances d'évaluation et de prospective de la recherche française. Ces statistiques sont également mises à la disposition des étudiants de second cycle.

Recherche au rabais : l'état paye 10.000 jeunes chercheurs au SMIC

16 La Confédération des Etudiants Chercheurs (CEC) lance un appel "Pour une revalorisation de l'allocation de recherche", à lire et à signer sur http://www.lapetition.com ou http:// www.lapetition.com/sign1.cfm?numero=381. Le montant de l'allocation de recherche, salaire octroyé par le ministère de l'Education Nationale à de nombreux doctorants, n'a pas été réévalué depuis 1991 et sera bientôt inférieur au SMIC.

17 La Confédération des Étudiants Chercheurs, fédération d'associations de doctorants visant à améliorer le statut des jeunes chercheurs français, défend l'idée que le doctorant (personne préparant un doctorat) est bien plus qu'un simple étudiant, comme le 
considèrent pourtant les institutions. Il est amené, durant sa thèse, à exercer toutes les activités d'un chercheur ou d'un enseignant-chercheur.

Elle condamne aussi l'aberration du discours gouvernemental qui souhaite valoriser la formation par la recherche tout en continuant de payer ses propres allocataires de recherche à un montant qui n'est plus qu'a $5 \%$ au dessus du SMIC. Cet état de fait est préjudiciable à l'ensemble des futurs docteurs, il dévalorise la formation doctorale et les activités de recherche de tous les doctorants, fragilisant ainsi leur insertion dans le secteur privé.

19 Afin de mettre fin a cette situation et de faire reconnaitre le caractère de professionnels à part entière des étudiants-chercheurs, la Confédération des Etudiants-Chercheurs a adressé au Premier Ministre, M. Lionel Jospin, une demande visant à revaloriser le montant de l'allocation de recherche au niveau qui était le sien en 1991 par rapport au SMIC, soit environ 9400 francs brut mensuels aujourd'hui.

Elle demande également que l'allocation de recherche entre dans le système d'indexation des salaires sur le point d'indice de la fonction publique.

21 Pour soutenir cette action, la Confédération des Étudiants-Chercheurs a lancé une pétition nationale dont le texte complet se trouve sur le site : http://www.lapetition.com/ sign1.cfm?numero=381, et appelle l'ensemble des personnes qui se sentent concernées par l'avenir de la recherche française.

22 La CEC souhaite plus généralement voir s'améliorer les conditions de financement de TOUS les doctorants, ainsi que leur accès à une protection sociale via un contrat de travail. L'ensemble de ses réflexions peut être consulté sur le site : http://garp.univbpclermont.fr/cec

Signez la pétition et participez ainsi à la reconnaissance des étudiants-chercheurs. 